\title{
The Investigations of Atrial Fibrillation Burden after Cryoballoon Ablation Detected by Insertable Cardiac Monitor in Patients with Paroxysmal Atrial Fibrillation: A Study Protocol for a Multicenter Randomized Controlled Trial (Cryo-ICM AF study)
}

Di Luo

the Second Hospital of Tianjin Medical University, Tianjin First Central Hospital https://orcid.org/00000002-9367-5319

\section{Yanhong Liu}

the second hospital of Tianjin medical university

yue zhang

the second hospital of Tianjin medical university

\section{Gang Xu}

the second hospital of Tianjin medical university

Enzhao Liu

the second hospital of Tianjin medical university

Tong Liu

the second hospital of Tianjin medical university

Guangping Li ( $\square$ tic_tjcardiol@126.com )

the second hospital of Tianjin medical university https://orcid.org/0000-0003-2121-2873

\section{Study protocol}

Keywords: Atrial fibrillation, Cryoballoon ablation, Randomized controlled trial, Insertable cardiac monitor

Posted Date: June 28th, 2021

DOI: https://doi.org/10.21203/rs.3.rs-434808/v1

License: (c) (i) This work is licensed under a Creative Commons Attribution 4.0 International License.

Read Full License 


\title{
The Investigations of Atrial Fibrillation Burden after Cryoballoon Ablation Detected by Insertable Cardiac Monitor in Patients with Paroxysmal Atrial Fibrillation: A Study Protocol for a Multicenter Randomized Controlled Trial (Cryo-ICM AF study)
}

\author{
Di Luo ${ }^{\# 1,2}$, Yanhong Liü ${ }^{\# 1,3}$, Yue Zhang ${ }^{1}$ Gang Xu${ }^{1}$, Enzhao Liư ${ }^{1}$, Tong Liu ${ }^{1}$, Guangping Li*1 \\ 1. Tianjin Key Laboratory of Ionic-Molecular Function of Cardiovascular disease, Department of \\ Cardiology, Tianjin Institute of Cardiology, the Second Hospital of Tianjin Medical University, Tianjin \\ 300211, China. 2. Tianjin First Central Hospital, Clinical Medical College of Tianjin Medical \\ University, Tianjin 300192, China. 3. The Third Central Hospital of Tianjin Medical University, Tianjin \\ 300170, China. \\ \# These authors contributed to the work equllly and should be regarded as co-first authors \\ *Correspondence: tic_tjcardiol@126.com
}

\section{Abstract}

Background: With the increasing number of aged population in China, the mortality and disability caused by atrial fibrillation (AF) and its complications lead to serious social burden and becoming more prominent. Due to the high false-negative rates of paroxysmal AF detected by routine ECG, the results on the recurrent rate and AF burden after cryoballoon ablation (CBA) in real world are inconsistent so far. Since the extension of monitoring time, the increased detection of AF in patients, insertable cardiac monitor (ICM) has its unique merits.

Methods: Cryo-ICM AF study is a prospective, multicenter randomized controlled trial with 200 participants with paroxysmal atrial fibrillation from 4 centers. Monitoring AF load after CBA though two monitoring methods - ICM and 24 hours holter during the 1 years follow-up of the two groups. All data will be observed and recorded in baseline, 30th days and $3^{\text {th }}, 6^{\text {th }}, 9^{\text {th }}$ and $12^{\text {th }}$ months after $\mathrm{CBA}$, which including incidence rates of postoperative complications and recurrence conditions, cardioelectrical indexes from 24-hours holter in all subjects and ICM in ICM group, UCG parameters about atrium/ventricular size and diastolic/systolic function, and biochemical indicators.

Disucssion: This is the first study design to confirm the effectiveness of cryoballoon in real world, monitoring AF load with ICM in patients with paroxysmal AF after CBA.

Trial registration: Chinese Clinical Trial Registry, No. ChiCTR1900026364. Registered 9 Oct 2019, http://www.chictr.org.cn/index.aspx

Availability of data and materials: Data supporting the results reported in this article can be found on the official website of Chinese clinical trials, http://www.chictr.org.cn/index.aspx.

Project Leader: Guangping Li*1, tic ticardiol@126.com,

Funding: the Second Hospital of Tianjin Medical University project (NO. 2019LC01, RMB 500,000). Science and technology project of Tianjin Health Committee (No. ZC20126). The project sponsor was NOT involved in the study design, analysis of data and the decision to submit the report.

Trial Status: Protocol Version number and Date: V1.0, 2019-05-30, the date recruitment began at 2020-06-23, the recruitment completed date is about at 2022-12-31.

Ethical approval: the ethics committee of the Second Hospital of Tianjin Medical University (Clinical ethical review, No. 2019-009)

Keywords: Atrial fibrillation, Cryoballoon ablation, Randomized controlled trial, Insertable cardiac monitor

\section{Background}

Atrial fibrillation (AF), the most common type of arrhythmia, occurs in patients with nonorganic or organic heart disease, such as hypertension, coronary heart disease, valvular heart disease and cardiomyopathy as well as apparent normal subjects. Wang et al ${ }^{[1]}$ used a recent national survey with stratified multistage random sampling from general Chinese population to estimate the AF disease burden, which enrolled 31,230 people aged 35 years or older all over China in the final analysis. The weighted prevalence of AF in the Chinese population aged 35 years or 
older was $0.71 \%$. The burden of AF in the general Chinese population was a significant public health issue in China. Epidemiologic investigations show the prevalence of $A F$ in natural population with different areas of China inland was an ascending trend with aging. The data in male and female under the age of 60 were $0.43 \%$ and $0.44 \%$ respectively, while over 60 years old were grown to $1.83 \%$ and $1.92 \%$ respectively. In population over than 80 years old, the number touched $7.5 \%$ [2-

3]. The risk of stroke, heart failure and dementia in patients with AF increased a 2-5 times, associated with a $40 \%-90 \%$ increased rate of mortality ${ }^{[4]}$. There should be a positive correlation between the occurrence of $A F$ and the growth of age. With the increasing number of aged population in China, the mortality and disability caused by AF and its complications lead to serious social burden and becoming more prominent.

Paroxysmal AF is difficult to be found because of the characteristics of concealment and atypical symptoms. However the external ambulatory electrocardiogram monitors have the demerit that the positive diagnosis rate of occasional and short-term arrhythmia was low. In this respect, we may say that the prevalence of paroxysmal AF in the real world is underestimated. Previous studies ${ }^{[5]}$ have suggested that continuous electrocardiographic monitoring increases the AF detection rate about 5.29 fold the odds than the baseline electrocardiography (ECG). The prevalence of $\mathrm{AF}$ using continuous electrocardiographic monitoring was $12.50 \%$ compared $2.26 \%$ using the routine strategy (24-hour Holter ECG and additional ECGs). On the other hand, the evidence goes to show that the extension of monitoring time, the increased detection of $A F$ in patients. Nevertheless with the extension of monitoring time, patients' compliance decreased. In addition, the detection rate of AF in routine ECG monitoring is not ideal. Due to the function of calculation method for AF (increased R-R interval stability for REVEAL XT and P-wave filtering for Reveal LINQ), insertable cardiac monitor (ICM) may offer clinical benefits for the sensitivity, specificity and accuracy in the diagnosis of $A F^{[6-7]}$. Taking these into account, ICM has its unique merits compared with the ambulatory electrocardiogram monitor ${ }^{[8]}$.

There is no denying that the treatment of AF has taken a huge leap forward in the last 30 years. STOP-AF ${ }^{[9]}$, a prospective randomized controlled multicenter trial, the result indicated that cryoballoon ablation (CBA) produced acute isolation of all four pulmonary veins (PVI) in $97.6 \%$ of patients, and treatment success was $69.9 \%$ compared with $7.3 \%$ of antiarrhythmic drug patients at 12 months follow-up. The STOP-AF trial demonstrated that cryoballoon ablation is a safe and effective alternative to antiarrhythmic medication for the treatment of patients with symptomatic paroxysmal AF. The FREEZE Cohort Study ${ }^{[10]}$ compares radiofrequency and cryoballoon ablation. The result reflects first-line ablation for paroxysmal AF is safe and effective with either radiofrequency or cryoballoon ablation, while the procedure time is significantly shorter in cryoballoon group. According to the result of STOP-AF and FREEZE, guidelines recommend CBA for patients who recurrent paroxysmal AF and failed therapy with antiarrhythmic drugs as I A recommendation. Considering the cost effect, the ICM instrument in this study we chose St.Jude Medical TM (SJM Confirm DM2102), manufactured by Abbott Company, USA and provided by Abbott, China.

So far the results on the recurrent rate, $A F$ burden and cardiac adverse events after $C A B$ are inconsistent, owe partially to the different detection methods of AF. To confirm the effectiveness of cryoballoon in real world, monitoring AF load with ICM in patients with paroxysmal AF after CBA: a multicenter randomized controlled trial will be conducted, with 200 participants from 4 centers.

\section{Methods/ design overview}

The study is designed as a prospective, randomized and multicenter study. The objective of the study is design to observe the recurrent rate of AF, the cardiac adverse events rate as well as the AF burden after CBA in the real world which will be evaluated by two monitoring methods: ICM and 24 hours holter during the 1 year's follow-up of the two groups. Also, it will explore the influence factors of recurrent AF after CBA using statistic method. Before the trial was conducted, informed consent will be obtained from all enrolled subjects. All study procedures were approved by the ethics committee of the Second Hospital of Tianjin Medical University (Clinical ethical review, No. 2019-009) as well as other participating medical centers, and all the centers could provide proof of laboratory quality control. The approval document which the ethical body approved our 
study is listed in Additional file 1. If there is any amendment to the protocol, approval must be sought again from the ethics committee. The study is registered with the Chinese Clinical Trial Registry (ChiCTR1900026364, Additional file 2). The training on the operation standard and recruit criteria of participants will be organized before the clinical trial start. All the selected subjects were randomly assigned to the ICM group or routine strategy (control group). Performance data will be collected for 12 months, followed by brief phone counseling, ECG analysis and/ or laboratory test and at 1 month and every 3 months after cryoballoon ablation.

\section{Enrollment}

Subjects who meet the inclusive criteria will be recruited from physicians, and randomized to the ICM group or control group using random number by Python programming software. The inclusive criteria are: (1) a history of nonvalvular paroxysmal AF confirmed by ECG or 24-hour Holter lasting for more than 30s, regardless duration, and consistent with radiofrequency ablation indications. (2) no previous ablation history. (3) no gender limitation and over 18. (4) all subjects are voluntary for the trial and willing to sign the informed consent form. (Additional file 3) The exclusion criteria are: (1) serious heart disease complicated with congestive heart failure (New York Heart Association grade III or IV ), arrhythmias and malignant hypertensive cause of hemodynamic instability. (2) valvular heart disease. (3) serious liver or renal dysfunction. (4) Abnormal state of coagulation or anticoagulant allergy. (5) Uncontrollable thyroid dysfunction. (6) mental illness has no independent capacity, or a possibility of being uncooperative in the followup period. (7) pregnancy or the possibility of pregnancy, or breast feeding. (8) Malignant tumor, life expectancy less than 1 year. Free post-operative $24 \mathrm{~h}$-holter examination and transportation fee subsidy can be provided for participating in this study.

\section{Methods/design}

The study is designed as a prospective, randomized and multicenter study to observe the recurrent rate of $A F$ after $C B A$, as well as the $A F$ burden and cardiac adverse events rate in the real world through two monitoring methods: ICM or Holter. Physicians screen and follow up the subjects in hospital outpatient or inpatient clinics. The Second Hospital of Tianjin medical university is the leader center (project protocol fund, Additional file 4), the sub centers of this study including, Tianjin Chest Hospital, the Second Hospital of Hebei medical university, Beijing Anzhen Hospital of Capital Medical University and the First Hospital of Peking university. Using PASS software to estimated the number of participants. The candidate subjects who meet the inclusion criteria and providing informed consent will be randomly assigned using random number by Python programming software into two groups (ICM or control group), by the School of Public Health of Tianjin Medical University, initially in a 1:1 ratio. The leader center will inform the random results according to the order of admission, and each sub center will compete for admission till enroll completed. The random results will be strictly confidential before the subjects signing the informed consent. All subjects' data will be collected using a standard case report form (Additional file 5) and transmitted to the central database at the data center. Missing or questionable data will be completed and corrected by queries. The subjects' information is confidential and all data will be used for this study. Baseline data will be collected including (1) sex, age, height, weight and BP. (2) all selected subjects will conventionally collected history (medication history of oral anticoagulant, history of smoking, AF combined with Type 2 diabetes mellitus, Hypertension or Cerebral vascular disease). (3) 24-hours Holter ECG for all subjects. (4) The blood samples will be test to measure the serum level of blood lipid and glucose, cardiac function and inflammatory index including the following indicators: Triglyceride (TG), Total cholesterol (TC), Low density lipoprotein cholesterol (LDL-C), High density lipoprotein cholesterol (HDL-c), creatinine ( $\mathrm{Cr}$ ), uric acid (UA), Troponin I (TNI), $\mathrm{N}$ terminal pro B type natriuretic peptide (NT-proBNP), glycosylated hemoglobin (HbA1c), fasting blood sugar (FBG), high-sensitivity C-reactive protein (hs-CRP) and complete blood count. (5) $\mathrm{CHA}_{2} \mathrm{DS}_{2}$-VASc score and body mass index (BMI) will be calculated. (6) The size of left atrium, left ventricular ejection fraction (EF) will be detected with ultrasound echocardiography (UCG). (7) Transesophageal echocardiography (TEE) applied to identify the diagnosis of thrombus in left atrium at pre-ablation.

All AF subjects undergo PVI through CBA, The subjects in ICM group implant ICM after the $\mathrm{CBA}$. The success rate of intra-operative each/total PVI, the time of PVI, the mean time of X-ray 
exposure, cryoballoon times and procedural time will be documented. Each pulmonary vein potential will be recorded by Achieve catheter before and after ablation. Record the ablation times, minimum temperature and the numbers of ablation of each pulmonary vein. Postoperative care for 24 hours by holter in each group.

Follow-up

Clinical follow-up using CRF will be performed at 30 days and every 3 months since then during the 1 year follow-up period. All data will be observed and recorded in 30 days $-3^{\text {th }} \neg 6^{\text {th }} \neg 9^{\text {th }}$ and $12^{\text {th }}$ months after CBA, which including incidence rates of postoperative complications and recurrence conditions, cardioelectrical indexes from 24-hours holter in all subjects and ICM in ICM group, UCG parameters about atrium/ventricular size and diastolic/systolic function, and biochemical indicators. The design of the study is shown in Fig 1. Remove the ICM equipment within one or three years after CBA depending on the patient demand. Install pacemaker when needed during the follow-up period is allowed.

Table 1 Participant timeline

\begin{tabular}{|c|c|c|c|c|c|c|}
\hline \multirow[b]{2}{*}{ Point time } & \multirow{2}{*}{$\begin{array}{l}\text { Enrollment } \\
\text { Baseline }\end{array}$} & \multicolumn{5}{|c|}{ Follow-up } \\
\hline & & 1 month & 3months & 6 months & 9months & 12 months \\
\hline Informed consent & $\sqrt{ }$ & & & & & \\
\hline General state & $\sqrt{ }$ & & & & & \\
\hline $\begin{array}{l}\text { laboratory } \\
\text { examination }\end{array}$ & $\sqrt{ }$ & & & & & $\sqrt{ }$ \\
\hline $24 \mathrm{H}-\mathrm{Holter}$ & $\sqrt{ }$ & $\sqrt{ }$ & $\sqrt{ }$ & $\sqrt{ }$ & $\sqrt{ }$ & $\sqrt{ }$ \\
\hline Echocardiography & $\sqrt{ }$ & & & & & $\sqrt{ }$ \\
\hline Operation record & $\sqrt{ }$ & & & & & \\
\hline $\begin{array}{l}\text { Postoperative } \\
\text { condition }\end{array}$ & $\sqrt{ }$ & $\sqrt{ }$ & $\sqrt{ }$ & $\sqrt{ }$ & $\sqrt{ }$ & $\sqrt{ }$ \\
\hline $\begin{array}{l}\text { ICM } \\
\text { programcontrolled }\end{array}$ & $\sqrt{ }$ & $\sqrt{ }$ & $\sqrt{ }$ & $\sqrt{ }$ & $\sqrt{ }$ & $\sqrt{ }$ \\
\hline Medication record & $\sqrt{ }$ & $\sqrt{ }$ & $\sqrt{ }$ & $\sqrt{ }$ & $\sqrt{ }$ & $\sqrt{ }$ \\
\hline
\end{tabular}




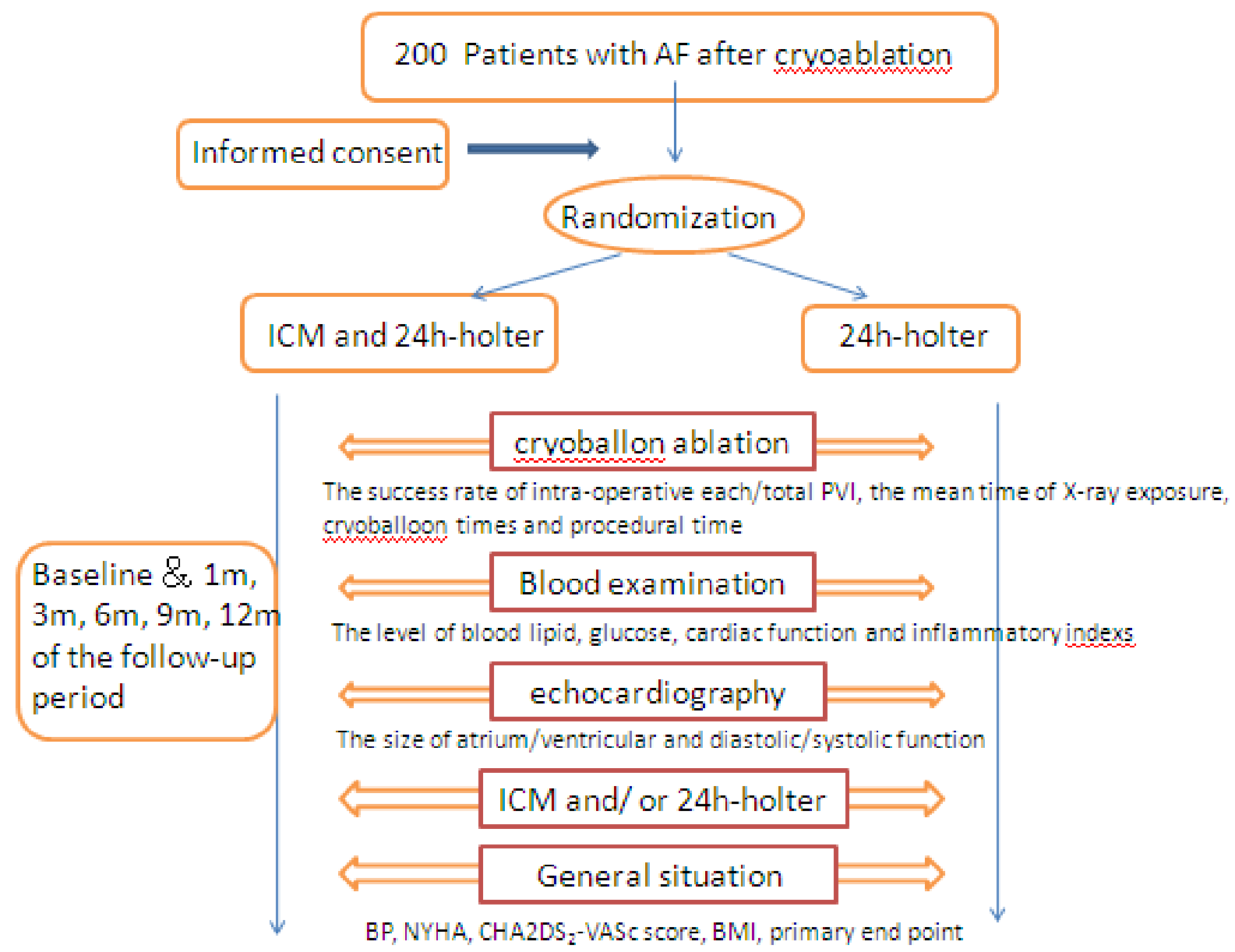

Fig 1 The technology roadmap of Cryo-ICM AF study

\section{Outcome measures}

The primary outcome measure is to observe the recurrent rate of $A F$, the cardiac adverse events rate as well as the AF burden after ablation by two monitoring methods: ICM and 24 hours holter during the 1 year's follow-up of the two groups. Any complications associated with implantation of ICM which need to recover by removing the device make the subject terminated the study. The secondary outcome measures of this study include the occurrence of: (1) the complication within 30 days after CBA, for instance, phrenic nerve pal, pericardial tamponade, left atrial-esophageal fistula, arterial-venous fistulas, pseudoaneurysm, arterial embolism, and pulmonary vein stenosis. (2) adverse cardiovascular events, including cardiopulmonary resuscitation, malignant arrhythmia, implanted with a pacemaker or implantable cardioverterdefibrillator, myocardial infarction, heart failure (NYHA III-IV), cardiogenic shock, pulmonary embolism, stroke/transient ischemic attack, cardiovascular mortality and all-cause death. (3) Any complications associated with implantation ICM. All the above contents will be recorded in Case report form. The management of complications (associated with implantation of ICM) will be provided by the recruited doctors, and the research funds responsible.

\section{Definitions}

The definitions of AF are made according to 2019 AHA/ACC/HRS for the management of patients with AF guidelines ${ }^{[11]}$. Any arrhythmia that has the ECG characteristics of AF and lasts sufficiently long for a 12-lead ECG to be recorded, or at least 30 seconds on a rhythm strip, should be considered an AF episode. The diagnosis requires an ECG or rhythm strip demonstrating: (1) "absolutely" irregular RR intervals (in the absence of complete AV block), (2) no distinct P waves on the surface ECG, and (3) an atrial cycle length (when visible) that is usually variable and less than $\mathbf{2 0 0}$ milliseconds. Paroxysmal AF is defined as recurrent AF ( $\geqslant$ two episodes) that terminates spontaneously or by doctor intervened within seven days. Acute procedural success is defined as 
complete electrical isolation of all pulmonary veins. Recurrent AF/AFL/AT is defined as AF/AFL/AT of at least 30 seconds' duration that is documented by an ECG or ICM and occurs following CBA. Recurrence of AF post ablation is defined as a recurrence of AF more than 3 months following AF ablation. Episodes of atrial tachycardia or atrial flutter should also be classified as a recurrence. A blanking period of three months should be employed after ablation when reporting.

\section{Data management and Analysis plan}

Baseline and follow-up information will be collected for each group through the questionnaire. A double data entry method will be used to ensure high quality of the data. Guangping Li as the leader of this project have access to the final trial dataset. The data monitoring committee (DMC) consists of relevant experts from the Second Hospital of Tianjin Medical University. To ensure the integrity of the study and the rights of the subjects, DMC will be independent from investigators, monitor every six months and put forward suggestions for revision. The data and results will be uploaded to Chinese clinical trial registry official website.

The analysis of variance or Kruskal-Wallis rank sum test will be used for continuous variables, and Chi-square tests will be used for categorical variables. Independent t-test or rank sum test will be used in comparing between experimental group and control group. Logistic regression analysis will be applied to identify clinical characteristics of subjects and cardioelectrical indexes associated with recurrence of AF. Kaplan-Meier curve for survival analysis and Cox regression to evaluating prognostic factors. All statistical analyses will be performed using SPSS statistical software (version 17.00, Chicago, IL, USA).

\section{Discussion}

Atrial fibrillation is one of the most common arrhythmia diseases. Embolism is a major complication of AF, especially ischemic stroke, which is an important risk factor of disability. As China now is facing the era of an aging population with fast speed, elder people demand to improve medical care and it has been a common focus for our whole society to solve. Research had showed that the elderly would be the main susceptible population of complications such as thromboembolism related to atrial fibrillation. With advancing age, the risk of thromboembolism and cardiac insufficiency in patients with AF is also increasing. To strengthen the management of AF can prevent disabling and even fatal complications, also can improve prognosis and the quality of life ${ }^{[12]}$. The recovery and maintenance of sinus rhythm can improve the prognosis of patients with AF, which can be achieve through long-term class I or class III antiarrhythmic drugs. However, previous clinical studies ${ }^{[9-10]}$ have shown that the effect of drugs on maintaining long-term sinus rhythm is unsatisfactory, and the side effects caused by long-term use of antiarrhythmic drugs will also reduce its benefits. As a non drug therapy, catheter ablation has been proved to be effective and safe for AF treatment. Catheter ablation is the first choice in the treatment of paroxysmal AF for whom is not effective or cannot tolerate the side effects of antiarrhythmic drugs( Guide ${ }^{\text {[13- }}$ ${ }^{14}$ ] Class IA indications).

$\mathrm{PVI}$ is the cornerstone of AF ablation strategy. However, the received ablation treatment rate among the patients who meet the indications of catheter ablation is extremely low. The application of point by point ablation is limited due to its own characteristics such as serious complications, complicated to operate and a long learning curve. The application of CBA in ablation of AF is an important technical breakthrough. The balloon can fit into the pulmonary vein ostia. This technique enables a "single shot" PVI by placing the balloon at the orifice of pulmonary veins, which can ablating circumferentially around the pulmonary veins ostia with energy. The CBA technique reduces the operating difficulty, shortens the operating time, had a short learning curve thus widely spread and applied in AF ablation.

As mentioned earlier, the STOP-AF ${ }^{[9]}$ and the FREEZE ${ }^{[10]}$ Study have confirmed that the therapeutic effect of cryoballoon on paroxysmal AF is no less than that of radiofrequency catheter ablation. The FIRE AND ICE trial ${ }^{[15]}$ is a prospective, randomized, controlled, open, blinded outcome assessment, non-inferiority trial comparing two different technologies: cryoballoon and radiofrequency ablation using 3-dimensional mapping to perform catheter ablation of PAF. It aims at providing objective data to guide selection and usage of ablation catheters in the treatment of AF. The immediate success rate was $98.9 \%$ in the CBA group and $97.9 \%$ in the radiofrequency group. After the 90 days blank period, the failure rate of 1-year survival curve in the CBA group was $34.6 \%$, 
and the rate in the radiofrequency group was $35.9 \%$. It is confirmed that the effectiveness of cryoablation is not inferior to radiofrequency ablation. RADICOOL Trial ${ }^{[16]}$ is a multicenter, retrospective study which examines the second-generation cryoballoon performance in a US multicenter review of real-world practices. The results showed that acute PVI was achieved in $99 \%$ of patients by cryoballoon catheter ablation. Average procedure time was 128 (range 82 to 260) min, using an average of 17 (range 1 to 19) min of fluoroscopy. There were no strokes, transient ischemic attacks, cardiac tamponade, atrioesophageal fistulas, or deaths during the study. At the 12-month efficacy endpoint, single-procedure success of freedom from atrial arrhythmia was $87 \%$. This real-world examination of the US practice demonstrates that second-generation cryoballoon ablation by PVI strategy is safe and effective among patients with paroxysmal AF. Some observational studies ${ }^{[17-19]}$ also confirm this conclusion.

It is important to screen AF because there are up to $40 \%$ of AF patients have no clinical manifestations, and about $25 \%$ of the first visits are due to stroke. Reveal AF [20], a multi-center, prospective long-term study has showed that among patients with a recent cryptogenic stroke or TIA who were 55 years or older, paroxysmal atrial fibrillation was common. ECG monitoring includes external ECG monitoring and cardiac implantable electronic-device. External ECG monitoring such as 24-hour ambulatory ECG and mobile ECG telemetry, has limited ability to diagnose incidental and short-term arrhythmias arrhythmia. With the extension of monitoring time, patients' compliance gradually decreased.

ICM can be continuously monitored and has no impact on patients' daily life. CRYSTAL AF Study ${ }^{[21]}$ and EMBRACE Trial ${ }^{[22]}$, all of these studies suggest that ICM is better than regular ECG in screening AF. Nowadays, ICM has been developed into equipment with small size and low power consumption, long battery life, simple implantation method, and compatibility with magnetic resonance inspection, which may have better application in the future. ICM of this study is manufactured by Abbott Company, USA and provided by Abbott, China. An ICM device goes under the skin and implanted in the fourth intercostal space on the left side of the anterior chest, corresponding to the position of ECG leads V2 and v3, which can monitor for up to two years. Placing Confirm $\mathrm{Rx}^{\mathrm{TM}}$ ICM is an easy procedure that takes very little time and the recovery is fast as well. The mobile app should be installed in order to send data to the doctor through smart phones.

Due to the high false-negative rates of AF detected by routine ECG, the results on the recurrent rate and $A F$ burden after $C A B$ in real world are inconsistent so far. To confirm the effectiveness of cryoballoon and monitoring AF load after CBA, two monitoring methods - ICM and 24 hours holter during the 1 years follow-up of the two groups will be conducted, which is a prospective, multicenter randomized controlled trial with 200 participants from 4 centers trial. The trial is currently in the recruitment phase.

\section{Declaration}

(1) Ethical approval: the ethics committee of the Second Hospital of Tianjin Medical University (Clinical ethical review, No. 2019-009)

(2) Consent for publication: This study will obtained the informed consent of all the subjects. All the personal data will be collected and statistics used for publication of the results of this study.

(3) Availability of data and materials: Data supporting the results reported in this article can be found on the official website of Chinese clinical trials, http://www.chictr.org.cn/index.aspx.

(4) Competing interests: The authors declare that they have no competing interests, and consent for publication. (5) Funding: the Second Hospital of Tianjin Medical University project (NO. 2019LC01, RMB 500,000). Science and technology project of Tianjin Health Committee (No. ZC20126). The project sponsor was NOT involved in the study design, analysis of data and the decision to submit the report. There is no economic relationship between all the researchers and the funding/competitors of this research project.

(6) Authors' contributions: Di Luo and Yanhong Liu participated in the study design, drafted the manuscript, planned the statistical analysis, and revised the manuscript. Yue Zhang and Tong Liu participated in the study conception and design, and contributed to revising the manuscript. Gang $\mathrm{Xu}$ and Enzhao Liu are in the diagnosis committee of ICM and 24 hours holter. Guangping Li as the principal investigator, conceived the study, dobtained ethical approval, and reviewed the manuscript. All authors read and approved the final manuscript. 


\section{Reference}

[1] Wang Z, Chen Z, Wang X, et al. The disease burden of atrial fibrillation in China from a national cross-sectional survey. Am J Cardiol, 2018, 122(5): 793-798. DOI: 10.1016/ j.amjcard.2018. 05.015.

[2] Zhou Z, Hu D. An epidemiological study on the prevalence of atrial fibrillation in the Chinese population of mainland China. J Epidemiol, 2008, 18(5): 209-216. DOI: 10.2188/jea.je2008021

[3] Zhang S. Atrial fibrillation in mainland China: epidemiology and current management. Heart, 2009, 95(13): 1052-1055. DOI: 10.1136/hrt.2008.146589.

[4] Reddy VY, Akehurst RL, Armstrong SO, et al. Time to cost- effectiveness following stroke reduction strategies in AF: warfarin versus NOACs versus LAA closure. J Am Coll Cardiol, 2015, 66(24): 2728-2739. DOI: 10.1016/j.jacc.2015.09.084.

[5] Suissa L, Lachaud S, Mahagne M H. Optimal timing and duration of continuous electrocardiographic monitoring fordetecting atrialfibrillation in stroke patients. J Stroke Cerebrovasc Dis, 2013, 22(7): 991-995. DOI: 10.1016/j.jstrokecerebrovasdis.2012.01.015

[6] Sanders P, Purerfellner H, Pokushalov E, et al. Performance of a new atrial fibrillation detection algorithm in a miniaturized insertable cardiac monitor: results from the Reveal LINQ Usability Study. Heart Rhythm, 2016, 13(7): 1425-1430. DOI: 10. 1016/j. hrthm. 2016. 03. 005.

[7] Purerfellner H, Pokushalov E, Sarkar S, et al. P-wave evidence as a method for improving algorithm to detect atrial fibrillation in insertable cardiac monitors. Heart Rhythm, 2014, 11(9): 1575-1583. DOI: 10.1016/j. hrthm. 2014. 06. 006.

[8] January CT, Wann LS, Calkins H, et a1. 2019 AHA/ACC/HRS focused update of the 2014 $\mathrm{AHA} / \mathrm{ACC} / \mathrm{HRS}$ guideline for the management of patients with atrial fibrillation. J Am Coll Cardiol. 2019, 74(1):104-132. DOI: 10.1016/j.jacc.2019.01.011.

[9] Packer DL, Kowal RC, Wheelan KR, et al. Cryoballoon ablation of pulmonary veins for paroxysmal atrial fibrillation: first results of the North American Arctic Front (STOP-AF) pivotal trial. J Am Coll Cardiol, 2013, 61(16): 1713-1723. DOI: 10.1016/j.jacc.2012.11.064

[10] Straube F, Dorwarth U, Ammar-Busch S, et al. First-line catheter ablation of paroxysmal atrial fibrillation: outcome of radiofrequency vs.cryoballoon pulmonary vein isolation. Europace, 2016, 18(3): 368-375. DOI: 10.1093/europace/euv271

[11] Craig T January, L Samuel Wann, Hugh Calkins, et al. 2019 AHA/ACC/HRS Focused Update of the 2014 AHA/ACC/HRS Guideline for the Management of Patients With Atrial Fibrillation: A Report of the American College of Cardiology/American Heart Association Task Force on Clinical Practice Guidelines and the Heart Rhythm Society in Collaboration With the Society of Thoracic Surgeons. Circulation, 2019, 140(2):e125-e151. DOI: 10.1161/CIR.0000000000000665.

[12] Giuseppe Boriani, Marco Proietti, Cécile Laroche, et.al. Contemporary stoke prevention strategies in 11096 European patients with atrial fibrillation: A Report From the EURObservational Research Programme on Atrial Fibrillation (EORP-AF) Long-Term General Registry. Europace, 2018,20(5):747-757. DOI: 10.1093/europace/eux301.

[13] Paulus Kirchhof, Stefano Benussi, Dipak Kotecha, et al. 2016 ESC Guidelines for the Management of Atrial Fibrillation Developed in Collaboration With EACTS. Rev Esp Cardiol (Engl Ed). 2017,70(1):50. DOI: 10.1016/j.rec.2016.11.033

[14] Calkins H, Kuck KH, Cappato R, Brugada J, Camm AJ, Chen SA, et al. 2012 HRS/EHRA/ECAS expert consensus statement on catheter and surgical ablation of atrial fibrillation: recommendations for patient selection, procedural techniques, patient management and followup, definitions, endpoints, and research trial design: a report of the Heart Rhythm Society (HRS) Task Force on Catheter and Surgical Ablation of Atrial Fibrillation. Heart Rhythm, 2012, 9: 632-96. DOI: 10.1016/j.hrthm.2011.12.016

[15] Alexander Fürnkranz, Josep Brugada, Jean-Paul Albenque, et al. Rationale and Design of FIRE AND ICE: A Multicenter Randomized Trial Comparing Efficacy and Safety of Pulmonary Vein Isolation Using a Cryoballoon Versus Radiofrequency Ablation With 3D-reconstruction. Cardiovasc Electrophysiol, 2014. 25(12): 1314-1320. DOI: 10.1111/jce.12529.

[16] Wilber Su, G Joseph Orme, Robert Hoyt, et al. Retrospective Review of Arctic Front Advance Cryoballoon Ablation: A Multicenter Examination of Second-Generation Cryoballoon (RADICOOL Trial). J Interv Card Electrophysiol, 51(3):199-204. DOI:10.1007/s10840-018-0335-1.

[17] Tian YL, Qi J, Wen QP, et al. Cryoballoon Ablation in Chinese Patients With Paroxysmal Atrial 
Fibrillation: 1-year Follow-Up. Pacing Clin Electrophysiol, 2017, 40(10): 1067-1072. DOI: 10.1111/pace.13157.

[18] Christian-Hendrik Heeger, Barbara Bellmann, Thomas Fink, et al. Efficacy and Safety of Cryoballoon Ablation in the Elderly: A Multicenter Study. Int J Cardiol, 2019,1(278):108-113. DOI: 10.1016/j.ijcard.2018.09.090.

[19] Ersan Akkaya, Alexander Berkowitsch, Sergej Zaltsberg, et al. Second-generation Cryoballoon Ablation for Treatment of Persistent Atrial Fibrillation: Three-year Outcome and Predictors of Recurrence After a Single Procedure. J Cardiovasc Electrophysiol. 2018, 29(1):38-45. DOI: 10.1111/jce.13372.

[20] David J Gladstone, Melanie Spring, Paul Dorian, et. Atrial Fibrillation in Patients With Cryptogenic Stroke. N Engl J Med, 2014, 370(26):2467-2477. DOI: 10.1056/NEJMoa1311376.

[21] Susan X Zhao, Paul D Ziegler, Michael H Crawford, et al. Evaluation of a Clinical Score for Predicting Atrial Fibrillation in Cryptogenic Stroke Patients With Insertable Cardiac Monitors: Results From the CRYSTAL AF Study. Ther Adv Neurol Disord, $2019,11: 12: 1756286419842698$. DOI: $10.1177 / 1756286419842698$.

[22] David J Gladstone, Paul Dorian, Melanie Spring, et al. Atrial Premature Beats Predict Atrial Fibrillation in Cryptogenic Stroke: Results From the EMBRACE Trial. Stroke, 2015, 46(4):936-941. DOI: 10.1161/STROKEAHA.115.008714. 


\section{Supplementary Files}

This is a list of supplementary files associated with this preprint. Click to download.

- 2.ChineseClinicalTrialRegisterChiCTR.pdf

- 5.CRF.pdf

- 6.LiSPIRITChecklistdownload8Jan13.pdf 